\title{
GREBI is a Novel Androgen-Regulated Gene Required for Prostate Cancer Growth
}

\author{
James M. Rae, ${ }^{1 *}$ Michael D. Johnson, ${ }^{2}$ Kevin E. Cordero, ${ }^{1}$ Joshua O. Scheys, ${ }^{1}$ \\ José M. Larios, ${ }^{1}$ Marco M. Gottardis, ${ }^{3}$ Kenneth J. Pienta, ${ }^{1}$ \\ and Marc E. Lippman ${ }^{1}$ \\ 'Division of Hematology Oncology, Department of Internal Medicine, University of Michigan Medical Center, \\ Ann Arbor, Michigan \\ ${ }^{2}$ Department of Oncology, Georgetown University, Washington, District of Columbia \\ ${ }^{3}$ Department of Discovery Biology, Bristol-Myers Squibb Pharmaceutical Research Institute, Princeton, New Jersey
}

\begin{abstract}
BACKGROUND. Gene regulated in breast cancer 1 (GREB1) is a novel estrogen-regulated gene shown to play a pivotal role in hormone-stimulated breast cancer growth. GREB1 is expressed in the prostate and its putative promoter contains potential androgen receptor (AR) response elements.

METHODS. We investigated the effects of androgens on GREB1 expression and its role in androgen-dependent prostate cancer growth.

RESULTS. Real-time PCR demonstrated high level GREB1 expression in benign prostatic hypertrophy (BPH), localized prostate cancer (L-PCa), and hormone refractory prostate cancer (HR-PCa). Androgen treatment of AR-positive prostate cancer cells induced dose-dependent GREB1 expression, which was blocked by anti-androgens. AR binding to the GREB1 promoter was confirmed by chromatin immunoprecipitation (ChIP) assays. Suppression of GREB1 by RNA interference blocked androgen-stimulated LNCaP cell proliferation.

CONCLUSIONS. GREB1 is expressed in proliferating prostatic tissue and prostate cancer, is regulated by androgens, and suppression of GREB1 blocks androgen-induced growth suggesting GREB1 may be critically involved in prostate cancer proliferation. Prostate

(C) 2006 Wiley-Liss, Inc.
\end{abstract}

KEY WORDS: $\quad$ prostate cancer; androgen receptor; androgen-induced growth; GREB1

\section{INTRODUCTION}

Prostate cancer is the most common lethal malignancy affecting men in the United States with an estimated 240,000 new cases and 30,000 deaths per year [1]. Androgenic hormones play a critical role in normal prostate function and have been shown to play a significant role in the both the initiation and progression of prostate cancer [2-4]. They mediate their physiological effects by binding to a specific steroid receptor, androgen receptor (AR), which is the key regulator of androgen signaling. Hormone bound AR forms homodimers which bind to androgen response elements (AREs) in gene regulatory regions and recruit coactivators which activate the transcription of androgen-regulated genes. The growth, development, and function of the prostate are controlled by androgens
Abbreviations: GREB1, gene regulated in breast cancer 1; AR, androgen receptor; $\mathrm{ER}$, estrogen receptor; $\mathrm{PR}$, progesterone receptor; $\mathrm{T}$, testosterone; DHT, $5 \alpha$-dihydrotestosterone; E2, $17 \beta$-estradiol; R1881, methyltrienolone.

Grant sponsor: Breast Cancer Research Foundation; Grant number: N003173; Grant sponsor: National Cancer Institute Specialized Program of Research Excellence in Prostate Cancer; Grant number: CA069568.

*Correspondence to: James M. Rae, Division of Hematology and Oncology, Department of Internal Medicine, University of Michigan Medical Center, 5323 Med Sci 1, 1150 W. Medical Center Drive, Ann Arbor, MI 48109. E-mail: jimmyrae@umich.edu

Received 21 September 2005; Accepted 8 December 2005

DOI 10.1002/pros.20403

Published online in Wiley InterScience

(www.interscience.wiley.com). 
through the complex pattern of genes regulated in this way, as is the proliferation of prostate tumors. Many androgen-regulated genes have the potential to regulate proliferation; however, the genes that are critically required for growth remain elusive. Identification of the key mediators of hormone-stimulated cancer growth could lead to new potential targets and more effective therapies.

Nearly all prostate cancers are initially $\mathrm{AR}+$ / androgen dependent and respond to anti-androgen therapy; however, almost all eventually develop an androgen-independent phenotype [4]. Deregulation of the AR signaling pathway plays a major role in the progression of prostate cancer to an androgen-independent/hormone refractory state and studies have shown that AR and most AR-regulated genes are constitutively expressed in the majority of androgenindependent tumors $[5,6]$. Thus, it seems likely that the key genes responsible for androgen-stimulated tumor cell proliferation become constitutively upregulated in androgen-independent disease and present attractive therapeutic targets.

In an attempt to identify key genes responsible for estrogen-stimulated proliferation in breast cancer, our laboratory used Affymetrix Genechip ${ }^{\mathbb{R}}$ microarrays to identify genes, the regulation of which mirrored the proliferative response of breast cancer cells to estrogens and anti-estrogens. Using this approach, we identified gene regulated in breast cancer 1 (GREB1), a gene previously known to be estrogen-regulated, and showed by small interfering RNA (siRNA)-mediated knockdown and other strategies that it is a key regulator of estrogen-stimulated proliferation [7]. Analysis of the $10 \mathrm{~kb}$ region $5^{\prime}$ of the GREB1 transcriptional start site demonstrated that it contains multiple putative hormone response elements including those for estrogen receptor (ER), progesterone receptor (PR), glucocorticoid receptor (GR), and AR. This led us to hypothesize that GREB1 might be an androgen-regulated gene, and that it could play an important role in androgen-regulated proliferation in prostate cancer.

\section{MATERIALS AND METHODS}

\section{Cell Lines, Culture Conditions, and Growth Assays}

Testosterone (T), dihydrotestosterone (DHT), 17 $\beta$ estradiol (E2), flutamide (Flu), nilutamide (Nil), and cyproterone acetate (Cyp-A) were purchased from Sigma-Aldrich, Inc. (St. Louis, MO). Bicalutamide $\left(\right.$ Casode ${ }^{\circledR}$ ) and methyltrienolone (R1881) were kindly provided by Dr. Diane M. Robins (University of Michigan, Ann Arbor, MI). Letrozole (Femara ${ }^{\circledR}$ ) was kindly provided by Dr. David A. Flockhart (Indiana University, Indianapolis, IN). Cell culture medium was purchased from Biosource International, Inc. (Camar- illo, CA) and bovine serums from Valley Biomedical, Inc. (Winchester, VA). All tumor cell lines were originally purchased from American Type Culture Collection (Rockville, MD) except the VCaP cell line which was obtained from the Pienta laboratory. Cell lines were maintained at $37^{\circ} \mathrm{C}$ in a $5 \% \mathrm{CO}_{2}$ atmosphere in minimal essential medium (IMEM) supplemented with $10 \%$ fetal bovine serum (FBS). For assays in defined hormone conditions, cells were washed and grown in steroid-depleted media (phenol red-free IMEM supplemented with $10 \%$ charcoal stripped calf bovine serum (CCS)) as described previously [7]. For growth assays, cells were plated in steroid-depleted media at $5 \times 10^{3}$ cells/well in 96-well plates (Falcon, Lincoln Park, NJ) and cultured for 3 days before treating with vehicle control (ethanol $0.1 \%$ ), E2, androgens, and the steroid antagonists. Relative cell number was determined using the crystal violet assay as described previously [8].

\section{Clinical Prostate Specimens}

Three benign prostatic hypertrophy (BPH), five localized prostate cancers (L-PCa), and seven hormone refractory prostate cancers (HR-PCa) (primary and metastatic) specimens were obtained from the University of Michigan Rapid Autopsy Tissue Procurement Program. Samples were collected with Institutional Review Board approval as described previously [9]. Clinical and pathology data were obtained as described previously [9].

\section{RNA Extraction}

Total RNA was isolated using TRIzol ${ }^{\circledR}$ Reagent (Invitrogen Corp., Carlsbad, CA) according to the manufacture's instructions. Yield and quality were determined by spectrophotometry (Beckman $\mathrm{DU}^{\mathbb{R}}{ }^{640}$, Beckman Coulter, Inc., Fullerton, CA) and using a Bioanalyzer RNA 6000 Nano chip (Agilent Technologies, Palo Alto, CA). All samples were stored at $-80^{\circ} \mathrm{C}$.

\section{Real-Time PCR}

GREB1 mRNA expression was measured using a semi-quantitative real-time PCR assay as described previously [7]. Briefly, total RNA $(1 \mu \mathrm{g})$ was reverse transcribed using Reverse Transcription System (Promega, Madison, WI) and cDNA amplified in a $25 \mu \mathrm{l}$ reaction containing Platinum Supermix UDG (Invitrogen Corp.), $250 \mathrm{nM}$ of each primer (forward 5'-CAA AGA ATA ACC TGT TGG CCC TGC- $3^{\prime}$ and reverse $5^{\prime}$ GAC ATG CCT GCG CTC TCA TAC TTA-3'-Integrated DNA Technologies, Inc., Coralville, IA), $10 \mathrm{nM}$ fluorescein (BioRad, Inc., Hercules, CA), and Syber Green. Reactions were performed using an iCycler Thermal Cycler (BioRad, Inc.). To control for RNA 
quality and quantity, GREB1 expression was normalized to the housekeeping genes 36B4 (forward 5'-GTG TTC GAC AAT GGC AGC AT-3' and reverse 5'-GAC ACC CTC CAG GAA GCG A-3') and GAPDH (forward 5'-GAA GGT GAA GGT CGG AGT C- $3^{\prime}$ and reverse 5'-GAA GAT GGT GAT GGG ATT TC-3') as described previously [7]. To evaluate the quality of product of real-time PCR assays, melt curve analyses were performed after each assay. Relative expression was determined using the $\Delta \Delta C_{T}$ method with either GAPDH or 36B4 as the reference genes [10].

\section{Chromatin Immunoprecipitation (ChIP) Assay}

LNCaP cells grown in steroid-depleted media were treated for 1 and $2 \mathrm{hr}$ with $10 \mathrm{nM}$ DHT or vehicle control. ChIP assays were performed using the QuickChIP Chromatin Immunoprecipitation Kit (IMGENEX Corp., San Diego, CA) according to manufacture's instructions with slight modifications. Chromatin was cross-linked using a $1 \%$ formaldehyde solution for $5 \mathrm{~min}$ at $37^{\circ} \mathrm{C}$ and sheared using the ChIP-IT Enzymatic Shearing Kit (Active Motif, Carlsbad, CA) for $10 \mathrm{~min}$. The samples were immunoprecipitated overnight at $4^{\circ} \mathrm{C}$ with $5 \mu \mathrm{g} / \mathrm{ml}$ of a polyclonal androgen-receptor antibody (catalog \#IMG-3238, IMGENEX Corp.), a goat IgG (catalog \#I5256, Sigma-Aldrich, Inc.) or a no antibody control. Washes, elution, and overnight reversal of cross-links were carried out according to the manufacturer's instructions. The DNA was purified using a QIAquick PCR Purification Kit (Qiagen, Inc., Valencia, CA) and PCR performed using an iCycler Thermal Cycler (BioRad, Inc.). Primers (forward sequence 5'-GGG AGG GAT GGA GGA CAA GAA G-3' and reverse 5'-GGC CAA CCC TCA CTC TAA CCC $C-3^{\prime}$ ) flanking the putative ARE from position -3371 to -3353 were used in three-step amplification reaction with an annealing temperature of $60^{\circ} \mathrm{C}$.

\section{GREBI mRNA Suppression Using siRNA}

Four synthetic siRNA duplexes were designed using standard criteria and purchased from Dharmacon, Inc. (Lafayette, CO). A non-silencing siRNA duplex, with no homology to any known sequence was used as a control (catalog \#D-001206-09, Dharmacon, Inc.). LNCaP cells were cultured for 4 days in steroid-free medium as described above, then transfected with $100 \mathrm{nM}$ siRNA specific to GREB1 or control siRNA using Oligofectamine ${ }^{\mathrm{TM}}$ reagent in serum-free OptiMEM-1 medium (Invitrogen Corp.) according to the manufacture's instructions for $4 \mathrm{hr}$ then medium replaced with phenol red-free IMEM supplemented with $10 \%$ CCS. After $24 \mathrm{hr}$, the cells were treated with R1881 $\left(10^{-9} \mathrm{M}\right)$ then assayed for GREB1 mRNA at $24 \mathrm{hr}$ and growth at 4 days.

\section{RESULTS}

Previous studies from our laboratory have shown that GREB1, an estrogen-regulated gene, is critically involved in hormone-dependent breast cancer growth. Sequence analysis of the putative GREB1 promoter region suggested that GREB1 might also be regulated by androgens and could play a similar key role in the regulation of prostate tumors by androgens. In order to evaluate this possibility, we first determined whether GREB1 is expressed at significant levels in prostatic tissue and in prostate tumors. RNA was prepared from three benign prostatic hypertrophy, five localized prostate cancer, and seven hormone refractory prostate cancer samples. GREB1 message levels were measured by real-time RT-PCR and the results are shown in Figure 1. GREB1 mRNA levels are expressed relative to the level in MCF-7 breast cancer cells which we have previously shown by real-time PCR and Northern blot analysis to express high levels of GREB1. Data are plotted on a log scale and all samples were found to express high levels of GREB1 consistent with the possibility that the gene plays an important role in prostate biology. GREB1 was also shown to be expressed in the AR-positive prostate cancer cell line $\mathrm{LNCaP}$, but not in the AR-negative line PC-3. This association with AR expression in cell lines was confirmed with additional AR-positive and negative lines $(\mathrm{VCaP}$ and DU-145, respectively; not shown), again suggesting that GREB1 might be an androgen-regulated gene.

In order to investigate the regulation of GREB1 expression by androgens, LNCaP cells were cultured in

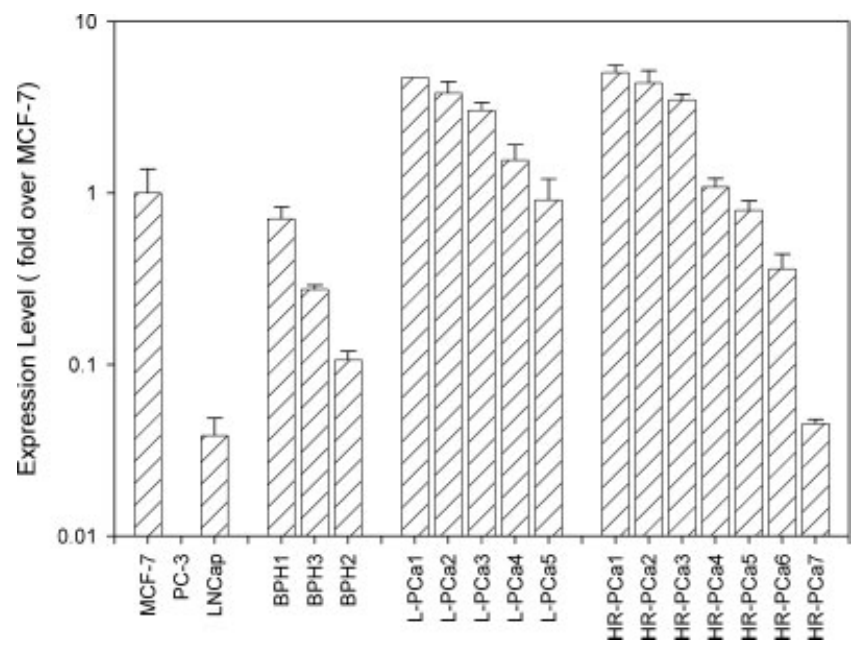

Fig. I. Clinical expression of GREBI. RNA was isolated from three benign prostatic hyper trophy (BPH), five localized prostate cancers (L-PCa), and seven hormone refractory prostate cancers (HR-PCa) (both primary and metastatic lesions) samples and assayed for GREBI mRNA expression using real-time RT-PCR. Results shown as the expression (mean and standard deviation of triplicate determinations) relative to expression level in MCF-7 breast cancer cells. 
steroid-depleted conditions then treated with the synthetic androgen R1881, or the natural AR ligands $\mathrm{T}$ and DHT $\left(10^{-12}\right.$ to $\left.10^{-8} \mathrm{M}\right)$. Representative data generated with LNCaP cells are shown in Figure 2a, with data plotted as the fold induction (mean and standard deviation of triplicate determinations) over vehicle control treated cells. All three androgens induced GREB1 mRNA in a dose-dependent manner with $10^{-8} \mathrm{M}$ R1881, T, and DHT inducing GREB1 by $7.3 \pm 1.2,9.8 \pm 1.2$, and $15.6 \pm 3.6$, respectively. Treatment with androgens produced very similar results in the $\mathrm{VCaP}$ cell line as shown in Figure $2 \mathrm{~b}$. We next tested whether the induction of GREB1 by androgens could be reversed with anti-androgens. In VCaP cells (Fig. 2c), $1 \mathrm{nM}$ T induced GREB1 (7.0 \pm 0.4 -fold) over control and was reversed by $1 \mu \mathrm{M}$ treatment of the anti-androgens bicalutamide ( $2.6 \pm 0.3$-fold), flutamide ( $3.6 \pm 0.9$-fold), nilutamide $(1.9 \pm 0.4)$, and cyproterone acetate $(3.6 \pm$ 0.9-fold). Bicalutamide, flutamide, and nilutamide alone (1 uM) did not affect expression of GREB1 while cyproterone acetate $(1 \mathrm{uM})$ alone did induce GREB1 (4.2 \pm 0.9 -fold) consistent with its known partial agonistic effects on the AR. Chromatin immunoprecipitation assay confirmed ligand-dependent AR binding to the putative ARE from position -3371 to -3353 as shown in Figure 3. LNCaP cells were grown in steroiddepleted conditions then treated with $10 \mathrm{nM}$ DHT for 1 and $2 \mathrm{hr}$. Immunoprecipitated DNA was readily detected after $2 \mathrm{hr}$ treatment while none was observed in the goat IgG controls and only trace amounts detected in the vehicle controls suggesting some residual androgens may have been present.

The LNCaP cells are androgen sensitive and their growth stimulated above basal levels when treated with androgens [11]. In our previous work, we showed that the estrogen-dependent proliferation of breast cancer cells could be suppressed by knocking down the expression of GREB1 with siRNA, suggesting that the gene plays a key role in estrogen-stimulated growth [7]. We were, therefore, interested in determining whether GREB1 might play a similar role in androgen-stimulated proliferation. $\mathrm{LNCaP}$ cells were cultured for 4 days in steroid hormone-free medium, then transfected with GREB1 siRNA duplexes. After $24 \mathrm{hr}$, the

Fig. 2. Dose-dependent induction of GREBI by androgens prostate cancer cell lines is reversible with anti-androgens. LNCaP (a) and $\mathrm{VCaP}(\mathbf{b})$ cells were cultured for 3 days in steroid-depleted conditions, treated with a dose range $\left(10^{-12}\right.$ to $\left.10^{-8} \mathrm{M}\right)$ of RI88I, T, and DHT. After $24 \mathrm{hr}$, cellular RNA was extracted and assayed for GREBI mRNA expression using real-time PCR. The effects of $\mathrm{T}\left(10^{-9} \mathrm{M}\right)$ were reversed when $\mathrm{VCaP}$ cells were cotreated $\left(10^{-6} \mathrm{M}\right)$ with antiandrogens CS, Flu, Nil, and Cyp-A (c). The results are shown as the fold normalized expression (mean and standard deviation of triplicate determinations) over vehicle control treated cells.
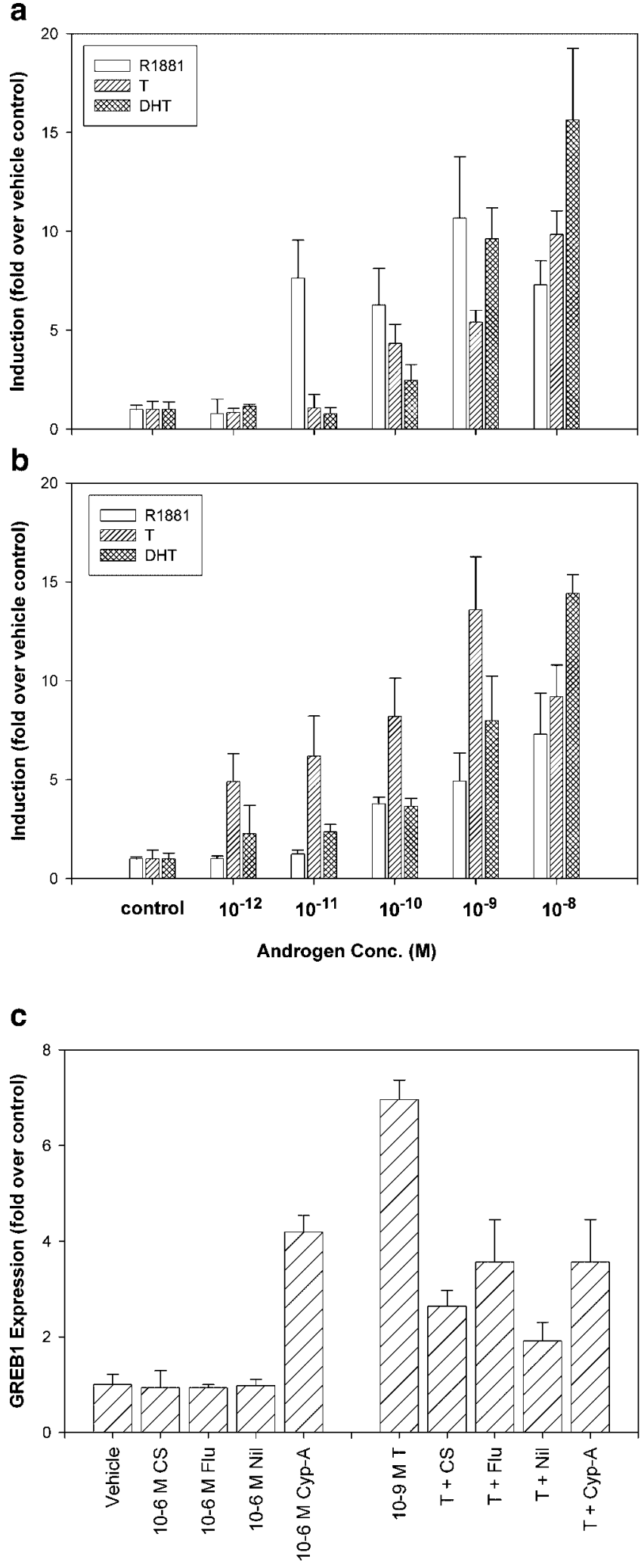


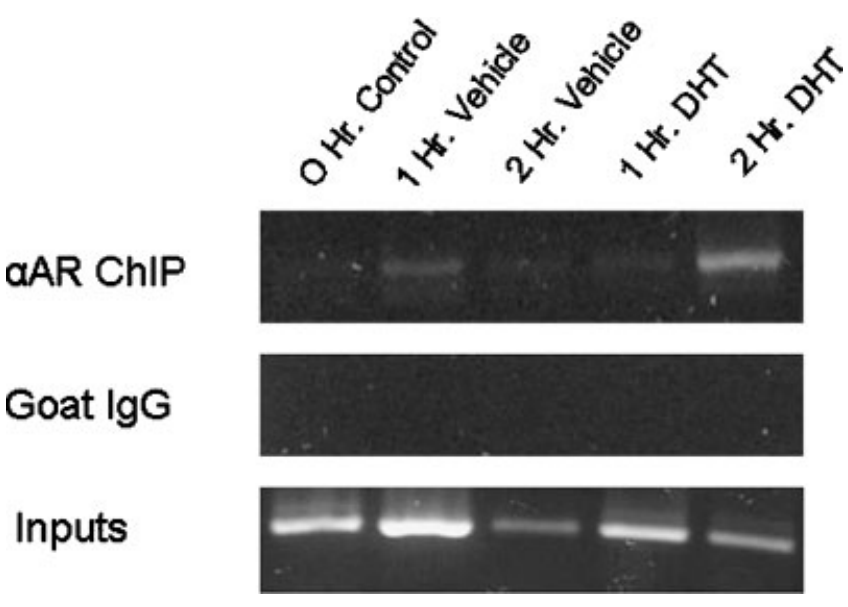

Fig. 3. Ligand-dependent $A R$ binding to a putative $A R E$ in the GREBI promoter. LNCaP cells were treated for I and $2 \mathrm{hr}$ with either vehicle control or $10 \mathrm{nM}$ DHT and ChIP assays performed as described in the Materials and Methods section. The putative ARE sequence was immunoprecipitated by an anti-AR antibody in extracts from cells treated for $2 \mathrm{hr}$ DHT. No signal was detected in the control antibody immunoprecipitates while control PCR reactions show robust amplification of the target sequence in input DNA.

cells were treated with $10^{-8} \mathrm{M}$ R1881, and then assayed for GREB1 expression and growth after $24 \mathrm{hr}$ and 4 days, respectively. As shown in Figure 4, treatment with $10^{-8}$ M R1881 induced GREB1 mRNA $16.4 \pm$ 5.7 -fold and cellular growth by $136 \pm 7 \%$. The control siRNA had no effect on either the basal or R1881induced GREB1 mRNA levels and growth. In contrast, the siRNAs targeting GREB1 blocked R1881-induced GREB1 mRNA induction by $59.5 \%$ and suppressed cellular growth to basal levels. These results suggest that GREB1 plays an important role in androgeninduced prostate cancer growth.

Previous studies have shown that LNCaP cells express a mutated form of AR which can be activated by estrogen [12]. The cells also express the $\beta$-form of the ER [13]. We wished to determine whether treatment with estrogen could also induce GREB1 in these cells. LNCaP cells were cultured in steroid-depleted conditions then treated with $10^{-9} \mathrm{M} \mathrm{E2}$ and assayed for GREB1 induction (Fig. 5a) and cellular growth (Fig. 5b). E2 treatment induced GREB1 mRNA by $11.6 \pm 5.4$-fold and cellular growth by $202 \pm 8 \%$. A 100 -fold molar excess of the anti-androgen bicalutamide or the antiestrogen ICI 182,780 partially blocked the effect of E2 while the combination of antagonist completely blocked the growth $(118 \pm 12 \%)$ and GREB1 induction $(0.5 \pm 0.7$-fold $)$ observed in these cells. No effect was seen with either bicalutamide or ICI 182,780 treatment in the absence of E2 (data not shown). These results show that both AR and ER $\beta$ can mediate GREB1
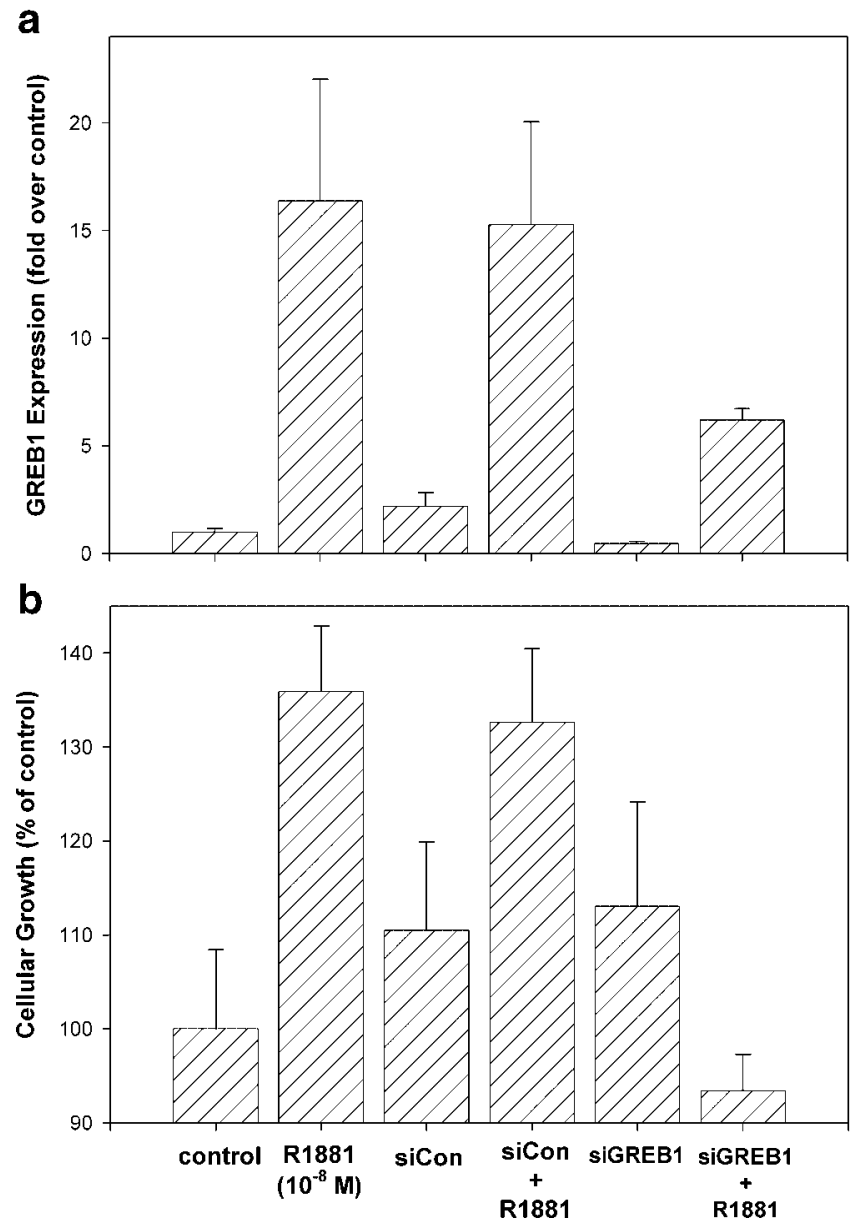

Fig. 4. Suppression of GREBI by siRNA blocks androgen-induced growth. LNCaP cells were cultured in steroid-depleted conditions, transfected with a pool of synthetic siRNA duplexes specific to GREBI or a control siRNA, and after $24 \mathrm{hr}$ treated with RI88I $\left(10^{-8} \mathrm{M}\right)$. a: After $24 \mathrm{hr}$, cellular RNA was extracted and assayed for GREBI mRNA using real-time RT-PCR and the results shown as the fold normalized expression (mean and standard deviation of triplicate determinations) over vehicle control treated cells. b: Cellular growth (\% over control) was assayed on day 4 using crystal violet staining and shown as the mean and standard error of six replicate samples.

induction in these cells and that GREB1 induction correlates with cellular growth.

To further study the induction of GREB1 expression by androgen and estrogen receptors, studies were done with a panel of cells of various AR and ER receptor status, using a variety of androgens and estrogens. The $\mathrm{ER} \alpha-/ \mathrm{AR}+$ breast cancer cell line MDA-MB-453 [14] showed a threefold induction of GREB1 when treated with $10^{-8} \mathrm{M}$ DHT. In contrast, neither R1881, T nor DHT treatment induced GREB1 in the ER $\alpha+/ A R-B T-$ 474 and T47D breast cancer cell lines despite robust induction by $\mathrm{E} 2$ (data not shown). Interestingly, we did observe a dose-dependent induction of GREB1 in the $\mathrm{ER} \alpha+/ \mathrm{AR}+\mathrm{MCF}-7$ breast cancer cell line when treated with $\mathrm{T}$ but not with R1881 at concentrations as high as 

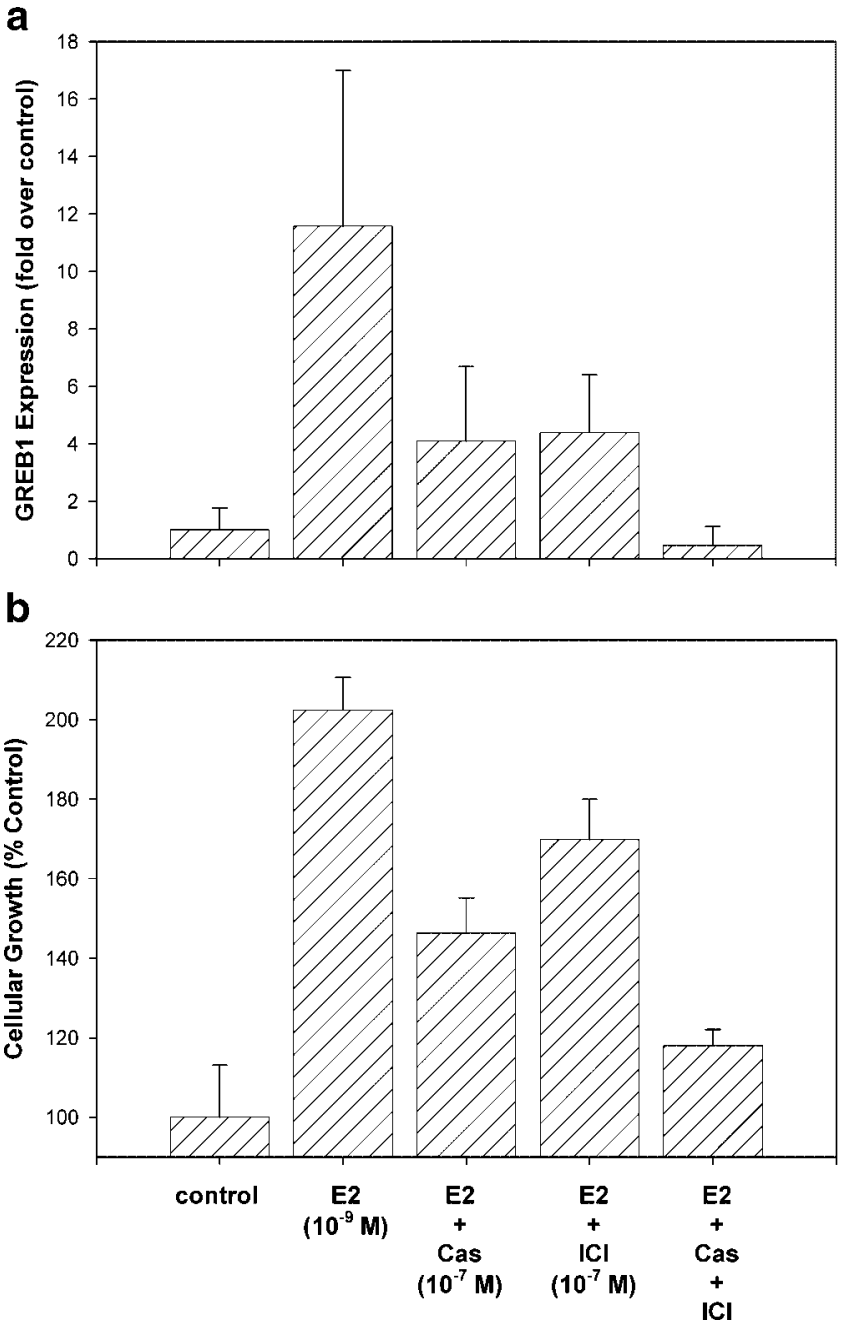

Fig. 5. Estrogen-induced GREBI expression in the $A R+/ E R \beta+$ $\mathrm{LNCaP}$ prostate cancer cell line. Cells were cultured for 3 days in steroid-depleted conditions before treatment with E2 ( $\left(0^{-9} \mathrm{M}\right)$, E2 plus bicalutamide $\left(10^{-7} \mathrm{M}\right)$, E2 plus $\mathrm{ICl} 182,780\left(10^{-7} \mathrm{M}\right)$ or E2 plus a combination of bicalutamide (Cas), and ICI 182,780 (ICl). a: After $24 \mathrm{hr}$, cellular RNA was extracted and assayed for GREBI mRNA using real-time RT-PCR and the results shown as the fold normalized expression (mean and standard deviation of triplicate determinations) over vehicle control treated cells. b: Cellular growth (\% over control) was assayed on day 4 using crystal violet staining and shown as the mean and standard error of 10 replicate samples.

$10^{-8}$ M. GREB1 is highly induced by E2 treatment in MCF-7 cells, and so we speculated that the observed GREB1 induction by $\mathrm{T}$ was due to aromatization of $\mathrm{T}$ to E2. To evaluate this possibility MCF-7 cells were treated with a range of concentrations of $\mathrm{T}\left(10^{-11}\right.$ to $\left.10^{-8} \mathrm{M}\right)$ and of the aromatase inhibitor letrozole $\left(10^{-7} \mathrm{M}\right)$. As shown in Figure 6, letrozole completely blocks Tmediated GREB1 mRNA induction (Fig. 6a) and growth (Fig. 6b) in MCF-7 cells while having no effect on E2-induced GREB1 mRNA (Fig. 6c) or growth (Fig. 6d) in these cells. In addition, the effects of T were completely reversed by treating with the anti-estrogen ICI 182,780 but not with the anti-androgen bicalutamide (data not shown). These results demonstrate that T-induced GREB1 mRNA and growth in MCF-7 cells is due to aromatization of $\mathrm{T}$ to $\mathrm{E} 2$.

\section{DISCUSSION}

Previously, we used DNA microarrays to identify estrogen-regulated genes in human breast cancer and found GREB1 to be one of only three genes induced by E2 that were common to three ER $\alpha+$ breast cancer cell lines. Our detailed in vitro and in vivo characterization of GREB1 demonstrated that it plays an important role in hormone-induced breast cancer growth [7]. In the current study, we show that GREB1 is regulated by the AR as well as ER $\beta$ and is required for androgeninduced prostate cancer growth. These results are not entirely surprising given the similarities between breast and prostate cancer including: high rates of hormone dependency, growth suppression with hormone blockade, and progression to hormone independency $[2,15]$. Although comparisons between ER- and AR-regulated genes have been made before, this is the first study to show that a critical regulator of hormoneinduced growth is share between breast and prostate cancer.

Ligand binding to AR induces a conformational change resulting in homodimer formation. These homodimers bind to AREs in gene promoter regions. There are numerous variants of the ARE consensus sequences found in multiple AR-regulated genes; however, they typically contain a core sequence of GGTACA nnn TGTTCT and require at least three out of four guanines (underlined) [16]. The putative GREB1 promoter region contains three potential AREs with one from position -3371 to -3353 having very similar sequence homology to the core sequence (gtGGTGCA tgg TGTTCAtc). ChIP analyses confirmed direct binding of $A R$ to this ARE in a ligand-dependent manner. These data clearly show that GREB1 is an androgenregulated gene as it is induced in a dose-dependent manner by multiple androgens and this is reversible with anti-androgens. Full analysis of the GREB1 promoter region is needed to confirm the identity and function of the AREs in the GREB1 promoter region.

In addition to androgens, $17 \beta$-estradiol induced GREB1 in the LNCaP cell line and this was partially reversed with anti-androgens and anti-estrogens and is completely reversed with a combination treatment. The LNCaP cell line was originally described as an AR+/ androgen-sensitive prostate cancer cell line established from a metastatic lesion [11]. Subsequent studies show that they express a mutated form of the androgen receptor that can bind and be activated by E2 as well 

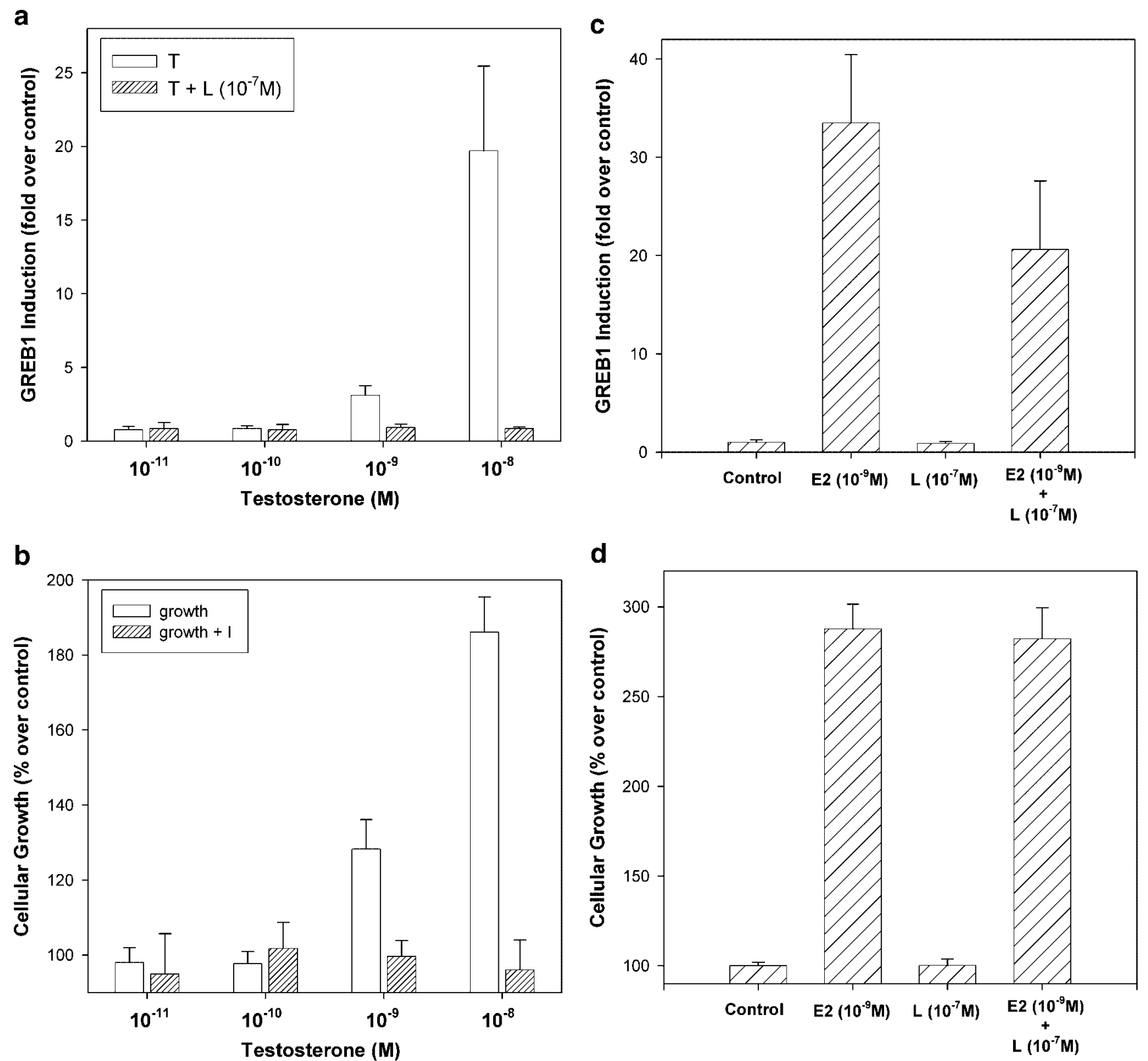

Fig. 6. Testosterone induces GREBI expression in the ER $\alpha+/ A R-M C F-7$ breast cancer cell line. Cells were cultured for 3 days in steroid-depleted conditions before treatment with a dose range of $\mathrm{T}\left(10^{-11}\right.$ to $\left.10^{-8} \mathrm{M}\right)$ either alone or in the presence of a fixed dose of letrozole $\left(10^{-7} \mathrm{M}\right)$. Shown as controls are the effects of letrozole $(\mathrm{L})$ alone and in combination with E2 $\left(10^{-9} \mathrm{M}\right)$. a and c: After $24 \mathrm{hr}$, cellular RNA was extracted and assayed for GREBI mRNA using real-time RT-PCR and the results shown as the fold normalized expression (mean and standard deviation of triplicate determinations) over vehicle control treated cells. $\mathbf{b}$ and $\mathbf{d}$ : Cellular growth (\% over control) was assayed on day 4 using crystal violet staining and shown as the mean and standard error of five replicate samples.

ER $\beta$ but not ER $\alpha$ [12,13]. We conclude that the induction of GREB1 by E2 in these cells is through both the mutated AR and ER $\beta$. Further support for this is that testosterone's effects on GREB1 expression could only be partially blocked by anti-androgens suggesting aromatization and activation of ER $\beta$. Although the induction of GREB1 through the androgen receptor pathway is a completely novel finding, the induction of GREB1 by ER $\beta$ has been shown previously. Stossi et al. investigated the differential gene regulation of $\mathrm{ER} \alpha$ versus ER $\beta$ by transfecting an ER-osteosarcoma cell lines with either isoform and identified genes using DNA microarray [17]. A search of the data presented in their study demonstrates that GREB1 is induced both by $E R \alpha$ and ER $\beta$ consistent with both our previous results concerning ER $\alpha$ [7] and our current results with ER $\beta$. We also noted that GREB1 promoter region contains progesterone receptor response elements. 
Although we did not test for progesterone receptor responsivity in this study, analysis of data presented by Jacobsen et al. using DNA microarrays and the human breast cancer cell line T47D engineered to express either $\mathrm{PR} \alpha$ or PR $\beta$ under an inducible promoter showed that GREB1 is induced by PR $\beta$ but not $P R \alpha$ in a classical ligand-dependent manner [18].

In all, it appears that GREB1 is regulated by multiple hormone nuclear receptors including at least $E R \alpha, E R \beta$, PR $\beta$, and AR as shown in this study. The significance of this is not yet understood; however, it does suggest that GREB1 plays an important role in hormone responsive tissue. GREB1 was originally discovered in the human brain [19]. Its gene spans roughly $108 \mathrm{~kb}$ of the short arm of chromosome 12 (p25.1). It has three known transcripts with the longest coding for a putative 1949aa protein with at least four transmembrane domains and a N-myristoylation domain (http://www.ncbi. nlm.nih.gov/IEB/Research/Acembly). Ghosh et al. were the first to show that GREB1 is an early response gene directly regulated by ER and suggested that it plays an important role in hormone-responsive tissues and cancer [20]. The cellular functional role of GREB1 is not yet known and is an area of active research. The results of this study are significant in that they provide an additional avenue for which to study the effects of GREB1 in hormone-stimulated cancer growth.

In this study, we found GREB1 to be highly expressed uniformly in both benign and malignant prostate cells and it is interesting to note that GREB1 was highly expressed in seven hormone refractory prostate samples taken from men who had bilateral orchiectomy and/or pharmacological blockade [9]. We did not observe any correlations between GREB1 expression and the pathology data available for these samples including Gleason score and AR status. This is probably due to the small number of samples analyzed and this important question is being addressed in future studies. However, it is interesting to note that GREB1 is expressed in AR-negative samples which may be due to ER $\beta$ activity in these cells. We therefore speculate that high expression of GREB1 plays a role in prostate cancer progression. Although we only examined a small number of clinical samples $(n=15)$, it appears that upregulation of GREB1 is a common occurrence in proliferating prostate tissues and suggest that it plays a role in prostate cancer growth. In support of this theory is the fact that deregulation of the androgen signaling pathway plays a major role in hormone refractory prostate cancer and that many of the genes required for androgen growth are upregulated [4]. Further evidence suggesting an important role for GREB1 in prostate cancer growth include our results using siRNA in which "knockdown" of the expression of GREB1 in the LNCaP cells led to the suppression of hormone-induced growth. Furthermore, these results are constant with what is known are about the differences between breast cancer and prostate cancers. Many breast cancers become hormone independent by upregulating alternative growth signaling pathways while prostate cancer tends to deregulate the AR signaling pathway in that the cells are no longer dependent upon hormone but are still dependent on the androgen signal transduction pathway.

In conclusion, our results show that GREB1 is an androgen-regulated gene, it is expressed in hormone refractory prostate cancer and suppression of GREB1 blocks androgen-induced growth. These results have important implications in that they suggest GREB1 may play an important role in prostate cancer growth and may be a prognostic as well as predictive marker of androgen responsiveness. Furthermore, our results suggest that GREB1 may be a target for prostate cancer treatment and suggested that the effects of GREB1 are not confined only to the breast but it also plays an important role in other hormone-dependent malignancies.

\section{REFERENCES}

1. American Cancer Society. Cancer Facts and Figures 2004. http://www.cancer.org/docroot/STT/stt_0.asp.

2. Lopez-Otin C, Diamandis EP. Breast and prostate cancer: An analysis of common epidemiological, genetic, and biochemical features. Endocr Rev 1998;19(4):365-396.

3. Jenster G. The role of the androgen receptor in the development and progression of prostate cancer. Semin Oncol 1999;26(4): 407-421.

4. Feldman BJ, Feldman D. The development of androgenindependent prostate cancer. Nat Rev Cancer 2001;1(1):34-45.

5. Bubendorf L, Kononen J, Koivisto P, Schraml P, Moch H, Gasser $\mathrm{TC}$, et al. Survey of gene amplifications during prostate cancer progression by high-throughout fluorescence in situ hybridization on tissue microarrays. Cancer Res 1999;59(4):803-806.

6. Linja MJ, Savinainen KJ, Saramaki OR, Tammela TL, Vessella RL, Visakorpi T. Amplification and overexpression of androgen receptor gene in hormone-refractory prostate cancer. Cancer Res 2001;61(9):3550-3555

7. Rae JM, Johnson MD, Scheys JO, Cordero KE, Laios JM, Lippman ME. GREB1 is a critical regulator of hormone dependent breast cancer growth. Breast Cancer Res Treat 2005;92(2):141-149.

8. Rae JM, Lippman ME. Evaluation of novel epidermal growth factor receptor tyrosine kinase inhibitors. Breast Cancer Res Treat 2004;83(2):99-107.

9. Shah RB, Mehra R, Chinnaiyan AM, Shen R, Ghosh D, Zhou M, et al. Androgen-independent prostate cancer is a heterogeneous group of diseases: Lessons from a rapid autopsy program. Cancer Res 2004;64(24):9209-9216.

10. Livak KJ, Schmittgen TD. Analysis of relative gene expression data using real-time quantitative PCR and the 2(-Delta Delta C(T)) method. Methods 2001;25(4):402-408.

11. Horoszewicz JS, Leong SS, Kawinski E, Karr JP, Rosenthal H, Chu TM, et al. LNCaP model of human prostatic carcinoma. Cancer Res 1983;43(4):1809-1818. 
12. Veldscholte J, Berrevoets CA, Brinkmann AO, Grootegoed JA, Mulder E. Anti-androgens and the mutated androgen receptor of LNCaP cells: Differential effects on binding affinity, heatshock protein interaction, and transcription activation. Biochemistry 1992;31(8):2393-2399.

13. Lau KM, LaSpina M, Long J, Ho SM. Expression of estrogen receptor (ER)-alpha and ER-beta in normal and malignant prostatic epithelial cells: Regulation by methylation and involvement in growth regulation. Cancer Res 2000;60(12):31753182.

14. Hall RE, Birrell SN, Tilley WD, Sutherland RL. MDA-MB-453, an androgen-responsive human breast carcinoma cell line with high level androgen receptor expression. Eur J Cancer 1994; 30A(4):484-490.

15. Coffey DS. Similarities of prostate and breast cancer: Evolution, diet, and estrogens. Urology 2001;57(4 Suppl 1): 31-38.

16. Nelson CC, Hendy SC, Shukin RJ, Cheng H, Bruchovsky N, Koop BF, et al. Determinants of DNA sequence specificity of the androgen, progesterone, and glucocorticoid receptors: Evidence for differential steroid receptor response elements. Mol Endocrinol 1999;13(12):2090-2107.

17. Stossi F, Barnett DH, Frasor J, Komm B, Lyttle CR, Katzenellenbogen BS. Transcriptional profiling of estrogen-regulated gene expression via estrogen receptor (ER) alpha or ERbeta in human osteosarcoma cells: Distinct and common target genes for these receptors. Endocrinology 2004;145(7):3473-3486.

18. Jacobsen BM, Schittone SA, Richer JK, Horwitz KB. Progesterone independent effects of human progesterone receptors (PR) in estrogen receptor positive breast cancer: PR isoform-specific gene regulation and tumor biology. Mol Endocrinol 2005; 19(3):574-587.

19. Nagase $T$, Ishikawa $K$, Miyajima $N$, Tanaka A, Kotani $H$, Nomura $\mathrm{N}$, et al. Prediction of the coding sequences of unidentified human genes. IX. The complete sequences of 100 new cDNA clones from brain which can code for large proteins in vitro. DNA Res 1998;5(1):31-39.

20. Ghosh MG, Thompson DA, Weigel RJ. PDZK1 and GREB1 are estrogen-regulated genes expressed in hormone-responsive breast cancer. Cancer Res 2000;60(22):6367-6375. 